\title{
¿Qué es PRogramar con orientación A objetos? Un enfopue fenomenográfico
}

Inés Friss de Kereki

\section{RESUMEN}

La enseñanza inicial de la programación está cambiando: de comenzar enseñando programación estructurada a comenzar con programación orientada a objetos. De a poco, los programas académicos de las universidades están encaminándose a esta -relativamente nueva- forma de programar. En 1996, en la Facultad de Ingeniería de la Universidad ORT Uruguay se realizó este cambio.

Este trabajo pretende investigar sobre cuál es la perspectiva de los alumnos sobre qué es la programación orientada a objetos, qué concepciones tienen y cuál es la utilidad futura que ellos sienten de este aprendizaje.

Con el objetivo de colaborar en la mejora de la enseñanza inicial de la programación proporcionando un aspecto diferente a los tradicionales y utilizando el método fenomenográfico de investigación, se realizaron entrevistas, se analizaron cuidadosamente y se arribó a un conjunto de categorías que representan concepciones cualitativamente diferentes del fenómeno estudiado.

\section{INTRODUCCIÓN}

En los programas académicos de Ingeniería en Sistemas, habitualmente se comienza enseñando programación utilizando técnicas de programación estructurada; pero la tendencia mundial es hacia la orientación a objetos. La mayoría de los programas de Ciencias de la Computación están incluyendo, desde los últimos 10 años, estos conceptos. En la Universidad ORT Uruguay, en la carrera de Ingeniería en Sistemas, comenzamos enseñando programación orientada a objetos. El lenguaje utilizado es Smalltalk V. Queremos estudiar cuál es la visión de los estudiantes que comienzan aprendiendo de esta manera.

¿Qué es programación estructurada y programación orientada a objetos?

El paradigma estructurado (o procedimental) de programación se basa en que la creación de un programa implica la definición de procesos que actúan sobre un conjunto independiente de datos.

La división entre datos y funciones o procesos impone una carga muy grande en el desarrollo del software. Las estructuras de datos (representación de la relación lógica entre elementos individuales de datos) requieren modificaciones con frecuencia y las funciones de control deben, en ese caso, ser examinadas de nuevo para ver si siguen estando coordinadas con los datos.

En el paradigma de objetos, el objetivo del diseño pasa de modelar el comportamiento del mundo a modelar objetos que existen en el mundo y sus comportamientos individuales. La arquitectura de la aplicación está, de esta manera, mucho más cerca de la estructura del problema.

El diseño orientado a objetos es una nueva forma de pensar los problemas usando modelos sobre conceptos del mundo real. Lo fundamental es construir el objeto, el cual combina estructuras de datos y comportamientos como una entidad única. 


\section{DEEINICIÓN DEL PROBLEMA}

\subsection{Antecedentes}

\subsubsection{SitUACIÓN ANTERIOR}

Desde hace varios años se dicta en la Universidad ORT Uruguay la carrera de Licenciatura en

Análisis de Sistemas. Entre sus objetivos figura el formar profesionales capaces de:

- identificar requerimientos organizacionales e informáticos que aumenten la competitividad y productividad de empresas y organizaciones;

- analizar, diseñar e implementar sistemas computacionales, reestructuras organizacionales y planes

de calidad e

- insertarse en el mercado nacional y regional como consultores, encargados de proyecto 0 gerentes

de sistemas.

Tiene una duración de 4 años. El requisito de ingreso es tener cursado y aprobado 6to. año de Bachillerato completo de cualquier orientación.

También existe la carrera de Analista Programad or. Su finalidad es, entre otras, formar profesionales en informática capaces de:

- insertarse rápidamente en el mercado laboral;

- asesorar a empresas como profesional independiente y

- adaptarse a los cambios tecnológicos.

Tiene una duración de 3 años y el requisito de ingreso es tener cursado y aprobado 4to. año de Secundaria completo.

Estas carreras han ido actualizándose. En 1995 se comenzaba enseñando programación estructurada utilizando Pascal. Los alumnos de la carrera Analista de Sistemas cursaban 4 semestres bajo el paradigma estructurado. En el quinto semestre se presentaba el paradigma de objetos.

El problema que veíamos era que, a esa altura, los alumnos no tenían capacidad de abstraerse de los temas básicos, es decir, ver los sistemas desde otra perspectiva diferente a la de cómo se programa.

Esto lo percibíamos en conversaciones informales con docentes y revisando trabajos producidos por los alumnos.

Hace algunos semestres, ya detectada esta dificultad y teniendo en cuenta también las tendencias de la programación, se hizo un primer intento de comenzar con la programación orientada a objetos. Se hizo este cambio en la carrera Analista Programador. Se comenzó con Pascal orientado a objetos. El resultado no fue lo esperado: los trabajos producidos por los alumnos no tenían, en general, un buen uso del paradigma. No se puede establecer en forma precisa las causas de este hecho, pero uno de los factores que suponemos influyó fue probablemente una inadecuada elección del lenguaje de programación. Se pretendió cambiar la forma de aprender a programar, pero no se cambió el lenguaje. Ese cambio además planteó dificultades porque había alumnos cursando la carrera con el plan anterior de la materia y con el plan nuevo.

\subsubsection{Situación actual}

En 1996 se creó la carrera de Ingeniería en Sistemas. Esta carrera tiene la finalidad de formar Ingenieros en Sistemas capaces, entre otras habilidades, de:

- diseñar y desarrollar sistemas de alta escala y complejidad;

- desempeñarse con éxito como desarrolladores de software, consultores independientes, líderes de proyectos o gerentes de sistemas y

- adaptarse al constante cambio en la industria e integrarse a equipos multidisciplinarios de investigación en el desarrollo de nuevas tecnologías.

En el currículo se incluyen materias vinculadas a bases de datos, diseño de sistemas, estructura de datos y algoritmos, ingeniería de software, programación y matemáticas. La carrera de Ingeniero 
Fue un excelente momento para empezar con el paradigma de objetos. No se crearían problemas con alumnos en diferentes etapas de la carrera, pues todos están comenzando.

Pocas universidades del mundo (pero cada vez más) están realizando experiencias de este tipo. Se relevó información vía Internet acerca de los programas de carreras similares de varias universidades y se confirmó que comienzan con el enfoque de programación estructurada.

Como elemento adicional, en el curso de Programación I y II se agregó una herramienta diferente a la práctica docente: el uso del TV Elite. En la clase se dispone de una computadora. EI TV Elite es un dispositivo que conecta el monitor de esa computadora con un televisor. De esta forma, el contenido de la pantalla de la computadora se ve también en el televisor. El software que acompaña al equipo ofrece opciones que permiten, entre otras funciones, resaltar áreas elegidas de la televisión, subrayarlas, ampliarlas.

Creímos necesario incluir este instrumento en la clase por varias razones. La razón principal fue que el lenguaje (Smalltalk) es de tipo visual. Esto es, muchas operaciones pueden ser realizadas directamente manipulando elementos de la pantalla. Por ejemplo, para cambiar el tamaño de un objeto en la pantalla es posible hacerlo modificando los valores numéricos de sus coordenadas 0 , en forma más sencilla, manipulando la propia imagen. Por esta causa, entendimos que disponer del equipo en clase para mostrar casos particulares y cómo se resuelven sería mucho más eficiente que contarles o dibujarles cómo se haría. Los alumnos verían en el momento cómo se hacen las cosas.

Otra razón fue que los estudiantes se podrían llevar a sus casas lo realizado en clase por medio de un diskette. No estarían 'apurados' por copiar los ejemplos de la computadora. Al final de la clase, realizarían su copia en un diskette permitiéndoles revisar y repasar lo hecho en clase en los laboratorios informáticos de la Universidad o en sus propias casas, ya que muchos estudiantes disponen de equipos propios.

Durante los cursos que dictamos, constatamos que el uso del TV Elite tuvo, además de las consideraciones anteriores, beneficios adicionales. Sentimos que los alumnos se interesaron mucho más en las clases porque tenían la respuesta a sus dudas dadas en la computadora en forma inmediata. Las preguntas del tipo, por ejemplo: '¿qué pasaría si....?' eran respondidas, además de la forma tradicional, mostránd oles en la computadora. Se vieron situaciones reales de prog ramación, con aciertos y errores. Esto brindó un aspecto de seguridad para los alumnos: 'no sólo lo dice el docente, yo lo vi'.

\subsection{OBjetivos}

El objetivo general de este trabajo es contribuir a mejorar, en la carrera de Ingeniería en Sistemas, los procesos de enseñanza y aprendizaje de la programación.

El objetivo específico es estudiar qué es programar con orientación a objetos y cómo aprenden los estudiantes de primer semestre en programación, y detectar características y tipologías esenciales.

La meta es determinar, si es que los hay, patrones de razonamiento que contribuyan a entender la programación.

Es importante resaltar que no es posible aún realizar estudios comparativos a largo plazo debido a que la carrera recién está comenzando.

\section{INVESTIGACIÓN}

\subsection{Método: Fenomenografía}

La monografía se realizó utilizando la tradición fenomenográfica de investigación. Este método de investigación cualitativa se ha desarrollado en la Universidad de Gotemburgo, Suecia.

La fenomenografía es el estudio empírico del número limitado de formas cualitativamente diferentes en las cuales los individuos experimentan, conceptualizan, entienden, perciben, aprenden, varios fenómenos y aspectos del mundo. Se trata de relevar, analizar y describir sistemáticamente esas formas cualitativamente diferentes. 
en Sistemas tiene una extensión de 5 años. El requisito de ingreso es haber cursado y aprobado 6 to. año de Ingeniería del Bachillerato Diversificado.

Por fenómeno entendemos algo del mundo, concreto o abstracto, que puede ser delimitado, por el investigador y otras personas, de acuerdo con su conocimiento del mundo. El fenomenóg rafo estudia la manera en la cual las personas entienden ese fenómeno, ap renden sobre él o experimentan con él. Una concepción es la relación cognitiva entre la persona y el fenómeno.

Una importante distinción para entender esta forma de investigación es la diferencia entre la perspectiva de primer y segundo orden. Cuando un investigador pregunta cuestiones sobre el mundo y mide sus resultados con una medida percibida o de verdad, se dice que se trabaja con la perspectiva de primer orden. Por ejemplo, ante la pregunta: '¿qué es morir?', el investigad or espera una respuesta basada en criterios biológicos o sicológicos. Es la perspectiva de primer orden. Si pregunta: '¿cómo las personas entienden la muerte?' o '¿qué piensa la gente que es la muerte?' es bastante diferente, requiere una perspectiva de segundo orden, interp retativa y espera una respuesta descriptiva y diferenciada: 'algunas personas piensan esto, otras personas piensan tal cosa y otras de tal manera'. No hay referencia a verdad o corrección.

No se trata de detallar las cosas como son, sino como ellas aparecen para las personas.

Para la colecta de datos se realizan entrevistas individuales. Las entrevistas, en general, pueden ser clasificadas en estructuradas 0 abiertas, pero este método utiliza la entrevista semiestructurada. En ella, el entrevistado es invitado a reflexionar sobre un determinado fenómeno a partir de preguntas generales. El énfasis está dado en captar el razonamiento y la perspectiva de la persona entrevistada. Las preguntas del entrevistador deben estar subordinadas a la consecución de tal fin. Se trata, en definitiva, de mantener un delicado equilibrio entre el respeto del discurso del entrevistado y las necesarias intervenciones del entrevistador. Las entrevistas son grabadas y transcriptas.

El resultado fundamental de la investigación fenomenográfica consiste en un conjunto o conjuntos de categorías. Cada categoría es nominada y se debe enunciar en la forma más pertinente y precisa posible. Luego se hace una distribución de frecuencias de las categorías.

En resumen, algunas características de este método son:

- es un método inductivo pues a partir de particularidades específicas se llega a conclusiones generales;

- el instrumento más utilizado es la entrevista semiestructurada y

- el resultado final de la investigación está dado por la obtención de un sistema de categorías, el cual se basa en una descripción analítica del material empírico.

\subsection{Colecta de datos de LA INVESTIGACIÓN}

La colecta de datos se realizó durante el período de setiembre a noviembre de 1996. Se trata de 20 entrevistas en profundidad que fueron realizadas en la Universidad ORT Uruguay, con una duración cada una de 20 a 30 minutos. Fueron grabadas y posteriormente transcriptas.

Todos los alumnos tienen el bachillerato terminado (6to. año). Aunque inicialmente pensamos que la mayoría de los alumnos no tendrían experiencia alguna en programación, constatamos que más del $80 \%$ de los entrevistados tenía experiencia: algunos con uno o dos cursos básicos, otros con varios años de trabajo en programación.

\subsection{ANÁlisis DE LAS ENTREVISTAS Y PRESENTACIÓN DE RESULTADOS}

Los alumnos tienen diferentes concepciones acerca de qué significa programar con un enfoque orientado a objetos. Del análisis del material empírico surgen las siguientes categorías:

\section{Categoría A:}

La programación orientada a objetos como medio de implementar programas en el computador. Los alumnos resaltan aspectos tales como la cantidad de cosas ya hechas y disponibles que se pueden utilizar, la preocupación por los detalles de implementación, y el ambiente (el lenguaje utilizado -Smalltalk- es más que un lenguaje: incluye un contexto entero en el cual trabajar, dando espacios de trabajo, editores y otras herramientas con las cuales crear un programa completo). Algunos destacan la dificultad de la sintaxis, es decir, los aspectos vinculados a la escritura del programa. Poder reutilizar 


\section{Categoría B:}

La programación orientada a objetos como un elemento potente y amplio para su futuro como profesional y como una herramienta informática. El énfasis en esta categoría se apoya en que se abren posibilidades para su futuro. Hay un interés en el futuro profesional y sienten la necesidad e importancia de la programación orientada a objetos, entre otros aspectos, por el poder que sienten que tiene y porque les simplifica el aprendizaje de más lenguajes. El aspecto central es su perspectiva de desarrollo profesional, en el que debe conocer esta herramienta como asunto relevante. Los individuos sienten que la programación orientada a objetos tiene poder y que forma parte de la tendencia en el desarrollo futuro de la programación. Finalmente destacan que les es de utilidad para trabajar en grupo 0 aprender más lenguajes.

\section{Categoría C:}

La programación orientada a objetos como una forma de entender, pensar y resolver problemas de la vida real. Los alumnos de esta categoría plantean que su forma de resolver problemas mediante la programación orientada a objetos, es tratando de ver la realidad del problema. El énfasis se coloca en la relación de la programación orientada a objetos y la resolución de problemas de situaciones concretas. En general, estos alumnos no implementan nada en la máquina hasta que tienen bien claro cuál es el problema a resolver y cómo resolverlo. Tampoco es visto como un problema dicha implementación. La preocupación mayor está puesta en el mundo concreto, es decir en la realidad. La programación orientada a objetos está al servicio de aquélla. Conciben la programación orientada a objetos como forma de entender, pensar y resolver problemas. Destacan la importancia de entender el problema, de saber qué es lo que hay que resolver antes de ver cómo se hace o se implementa. Es fundamental para ellos entender la situación.

\section{Distribución CUANTITATIVA:}

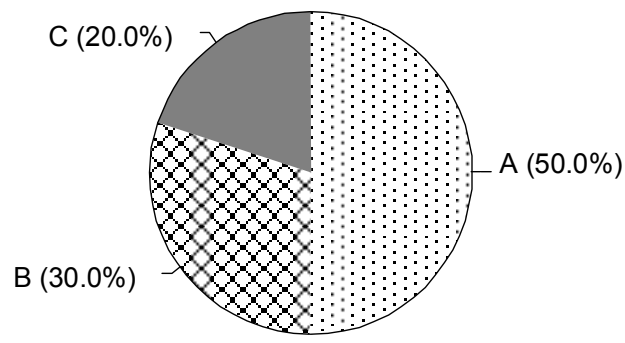

\section{Conclusiones}

Los alumnos entrevistados entienden la programación orientada a objetos de diferente manera, según lo presentado en el análisis empírico:

A. La programación orientada a objetos como medio de implementar programas en el computador; B. La programación orientada a objetos como un elemento potente y amplio para su futuro profesional y como una herramienta informática;

C.La programación orientada a objetos como una forma de entender, pensar y resolver problemas de la vida real.

Al analizar las categorías presentadas precedentemente, a la luz de un eje temporal, aparecen dos perspectivas diferentes: una orientada hacia el aquí y ahora y otra hacia el futuro. 
cosas, la preocupación por la inicialización de variables o detalles de la implementación, modificar en un solo lugar cuando sea necesario realizar algún cambio, el interés por investigar y averiguar cómo funcionan determinadas clases, son algunas de las áreas destacadas por estos alumnos.

\section{PeRSPECTIVA DEL AHORA}

Los estudiantes de las categorías A (la programación orientada a objetos como medio de implementar programas en el computad or) y C (la programación orientada a objetos como una forma de entender, pensar y resolver problemas de la vida real) están preocupados por la situación actual, por resolver problemas ahora sin tener en cuenta la proyección futura de su proceso de aprendizaje.

Parecería que, para ellos, el aprendizaje de la programación orientada a objetos es una cuestión concreta, puntual. No se percibe un razonamiento profundo sobre el significado de sus ap rendizajes ni de su utilidad a largo plazo. Los sujetos están en un curso que ha sido impuesto y no se cuestionan el por qué de esta enseñanza.

Los estudiantes razonan en torno a la programación orientada a objetos como algo relacionado con el aprendizaje en el mundo universitario. Su discurso no trasciende el espacio físico del aula y los laboratorios.

\section{Perspectiva del Futuro}

Los estudiantes de la categoría B (la programación orientada a objetos como un elemento potente y amplio para su futuro como profesional y como una herramienta informática) presentan un razonamiento articulado y consistente, y relacionan el aquí y ahora con su futura inserción laboral.

El aprendizaje de la programación orientada a objetos es vista en relación con el escenario de la vida de sus tareas profesionales. Los estudiantes están muy preocupados de que su aprendizaje sea un elemento que les permita actuar con efectividad y eficiencia fuera del ámbito universitario.

Los estudiantes relacionan el período de formación que desarrollan en el presente con su futuro rol como profesionales y como personas. Los alumnos, en definitiva, tienen claro el por qué y para qué de este aprendizaje.

\section{Bibliografía}

BOOTH,S.(1992) Learning to Program. A phenomenographic perspective. Gotemburgo. Universidad de Gotemburgo.

NAGLE,A. (1988) Orientación vocacional. Una investigación de la perspectiva de los jóvenes uruguayos. Gotemburgo. Universidad de Gotemburgo.

PRESSMAN,R.(1992) Ingeniería del Software. Un enfoque práctico. 2da. ed. México, Mc. Graw Hill

WINBLAD,A;EDWARDS,S y KING,D. (1993) Software orientado a objetos. Addison- Wesley Iberoamericana. 